\title{
Numerical analyses of adhesive-bonded joints in steel I-beams reinforced with CFRP strips
}

\author{
Maciej Kowal ${ }^{1, *}$, and Mateusz Hypki ${ }^{1}$ \\ ${ }^{1}$ Lublin University of Technology, Faculty of Civil Engineering and Architecture, Poland
}

\begin{abstract}
Numerical analyses represent a useful structure-dimensioning tool and are indispensable for the analyses of structures featuring varied materials or complex geometric layouts. Modelling reinforced structures by gluing their elements is complicated as frequently at least two elements, made of different materials, are joined. This influences their behaviour under a load. Numerical analyses can provide invaluable support in this respect. To compare the laboratory test results obtained by the authors referring to reinforcing bent steel I-beams with adhesive-bonded composite-CFRP strips, and theoretical analyses (which are not the subject of this study), a preliminary FEM analysis was carried out. FEM analysis models were created with the same geometries and material parameters of the strengthening composite strip, the adhesive, and the reinforced I-beam, as in the laboratory tests. The distribution of stresses along the bond end, and their maximum values at the strip end, were specified in detail. ABAQUS®6.12-3 software was used to verify the results. The compliance of the FEM analysis with the laboratory measurements was confirmed in terms of the identified values and shearing-stress diagrams.
\end{abstract}

\section{Introduction}

Designing structural reinforcements for bridges is a difficult yet crucial aspect of engineering. Using conventional methods of strengthening steel structures (cutting out and inserting a new element, increasing structure sections, external compression, or using ropeexpansion structures) can turn out to be cost-ineffective or technically difficult to perform. The structure's deadweight can be considerably increased in the process, and static patterns can be disrupted. The reinforcing structure can be difficult to apply, as well as susceptible to corrosion and wear; therefore, the result may not be completely satisfying.

Using CFRP (Carbon Fibre Reinforced Polymer) composites is a promising alternative to the currently used methods of reinforcing steel structures. CRRP composites are resistant to corrosion, and have low density and very high tensile strength. Additionally, the method of adhesive-bonding of composite elements is easy to apply.

Aerospace engineering is a pioneer in composite application. Mainly, concrete structures are reinforced with composites. However, the number of applications of composite strips to increase the bearing capacity of steel bridges is also on the rise. In Poland the bridge over the Vistula in Chełmno was reinforced using this method [1]. Elsewhere in Europe, mainly in the United Kingdom, the number of reinforcements of bridges with composites is more common, and focuses mainly on metal structures [2-6].
The major purpose of the article was to compare laboratory test results, obtained referring to bent steel Ibeams reinforced, with adhesive-bonded compositeCFRP strips with the FEM analysis results.

\section{Reinforcing steel structures with FRP composites in the literature}

An important stage in reinforcing steel structures with composite materials is preparing the contact surfaces of the reinforced element and the adhesive-bonded element for joining, and determining stresses in the adhesive layer. The strength of the joint has a considerable impact on the bearing capacity of the entire reinforcement system. The significance of this stage for structure reinforcement is described, i.a., in [7-11]. Exceeding border stresses in the adhesive, causing the disjoining of the reinforcement at the steel-adhesive bond, is the main, but not the only feasible form of damage to the structure.

Scientists, dealing with the reinforcement of steel structures with composite materials, seek solutions enabling a possibly permanent reinforcement with high efficiency. For this purpose, various solutions are considered for increasing the strength of the joint [12-14].

Reinforcing steel elements with FRP composites aims to restore or increase the structure's bearing capacity. In bridge structures, the main structural elements are usually the main girder bars. The steel bar tests to be found in the literature on the subject usually

\footnotetext{
* Corresponding author: $\underline{\text { m.kowal@pollub.pl }}$
} 
present three- or four-point bending bars with an I-beam [15-19].

\section{The laboratory tests presented by the authors}

The authors carried out tests at the Civil Engineering Laboratory of the Lublin University of Technology. The main objective was to confirm the possibility of increasing the strength of steel structure reinforced with adhesive-bonded composite-CFRP strips.

The tests were divided into three stages. In the first stage, various geometries of the end of the strip and the adhesive bond at the joint were compared. 78 samples were subjected to axial tension. Each sample consisted of two flat steel bars joined with carbon plates with specified dimensions, adhesive-bonded on both sides. The second stage consisted of verifying the results of the first. For this purpose, 18 reinforced samples were prepared with three types of strip ends, and subjected to three-point bending. The third stage tested the influence of composite-strip adhesion on increasing the bearing capacity of reinforced I-beams. Adhesives with three degrees of thickness $(0,6 \mathrm{~mm} ; 1,2 \mathrm{~mm} ; 1,8 \mathrm{~mm})$ and three anchoring lengths were considered $(65 \mathrm{~mm}, 165 \mathrm{~mm}$, $265 \mathrm{~mm}$ ), composite strips with two varying elastic moduli (168GPa and $210 \mathrm{GPa}$ ). In the third stage of the tests, undamaged I-beams were used - 3 for comparison, and 16 reinforced steel I-beams INP 140 with a theoretical span of $1.90 \mathrm{~m}$ - to perform 4-point bending. Two types of joints were checked -- the square end (square) and the one providing the best possible strength of the bond end selected from the ones used in the first stage, i.e. reverse tapered with an adhesive fillet (reverse tapered). Fig. 3.1 presents an outline of the sample with the load. More about the laboratory tests in [20].

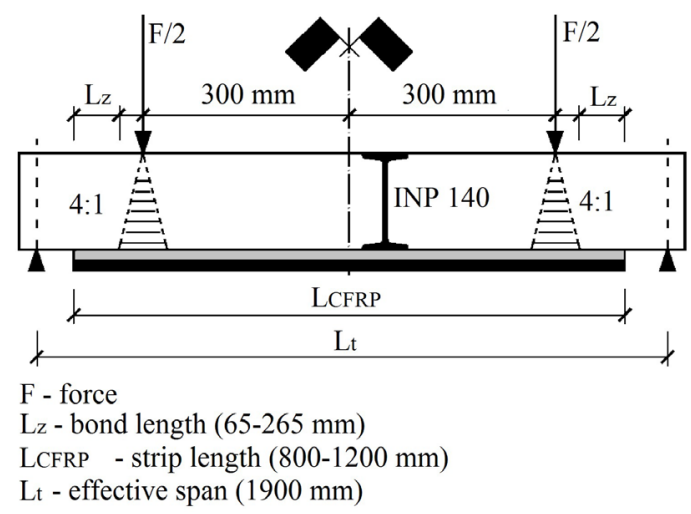

Fig. 3.1. The sample in the second stage of tests

All the reinforced samples were covered with electric-resistance tensometers. The adhesion patterns were changed depending on the type of the samples. Samples 200.120.Z.A (square, 1,2m long strip, 210GPa elastic module) and 200.120.F.A (reverse tapered, 1,2m long strip, $210 \mathrm{GPa}$ elastic module) were covered with nineteen tensometers arranged symmetrically around the centre of the span, as in the Fig. 3.2. In the case of these samples, Tenmex foil electric resistance tensometers type TFs10/120 were used, with a resistance of $120 \pm 0.2 \% \Omega$.

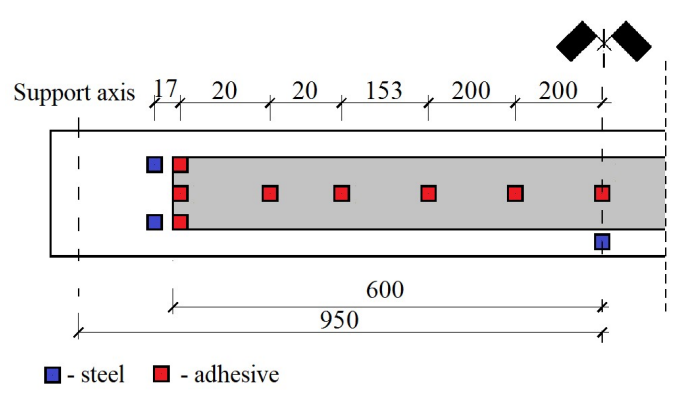

Fig. 3.2. Diagram of the distribution of tensometers for samples 200.120.F.A and 200.120.Z.A.

The 4-point bending tests were stopped after tearing the composite strip or reaching the force of $95 \mathrm{kN}$ on the press stroke, which ensured the plastic softening of the steel I-beam reinforced with the CFRP strip in the area where the forces concentrated on the upper flange were applied. The forces damaging the non-reinforced steel Ibeams oscillated around $76 \mathrm{kN}$.

Two main forms of sample damage were observed. The first was the disjoining of the composite strip at the steel-adhesive bond. This form was observed in 12 samples. The second observed form of damage was the plastic softening of the I-beam's steel, not accompanied by strip disjoining.

\section{Numerical analysis}

Numerical analyses are useful structure dimensioning tools, indispensable for analyses of structures with complicated geometric layouts or using varied materials. Depending on the purpose of the reinforced structure, the proper selection of the reinforced material dimensions, the elastic moduli, and the strength of the reinforcing material and the adhesive, are of key importance to the durability and strength of the reinforcement. A FEM analysis can bring invaluable support in this respect.

Among a broad range of numerical analyses described in the literature on the subject those dealing with the FEM models of steel beams reinforced with composite strips are few in comparison to other types of supports of steel elements with composites, i.a., [21-25]. The studies involve the verification of theoretical analyses of stresses at the bond end for various types of load on the beams.

To compare the obtained results of the authors' laboratory tests with the theoretical analysis, a preliminary MES analysis had been conducted. The tests were carried out to verify the accuracy of the developed theoretical model for determining shearing and peel stress, which is not the subject of this study. The secondary goal was to verify the shearing stress model against the laboratory test results. Almost all the experimental measurements of stresses described in the 
literature refer to shearing stresses and are based on the measurement of strains on the outer surface of the adhesive. The calculation of shearing stress at the bond end is based on the difference in strains between two neighbouring tensometric sensors, the values depending on the distance between the sensors and the thickness and the elastic modulus of the composite tape. This leads to including in the measurements at least two obvious approximate stress values. First, due to neglecting the impact of strip bending on the stress value, which is important in the strip end area. Second, the shearing stress between the adjacent tensometric sensors is averaged, and hence high shearing stress values in the strip end area might not be recorded. Furthermore, the average shearing stresses obtained because of such a measurement are closer to the stress values at the stripadhesive bond than to those of the adhesive-steel bond, which is more prone to disjoining. Similar conclusions can be found, i.a., in [22]. The authors are not familiar with any existing peel stress measurement methods.

\subsection{Numerical mode}

FEM analysis models were created with the same geometries and reinforcement parameters as the samples used in the laboratory tests. ABAQUS®6.12-3 software was used to verify the results. A three-dimensional model was created for a two-dimensional space, using shell-type elements, and taking into consideration the entire length of the bar. The attachment points at the end of the bar represented the support conditions in the vertical direction. A measurement grid for the beam model is presented in the Fig. 4.1. The steel bar, the adhesive, and the composite strip were modelled with 4 node doubly curved shell elements without the reduction of integration points (S4). To obtain accurate results for stresses in the strip end area, the grid was condensed in this area.

a) square end

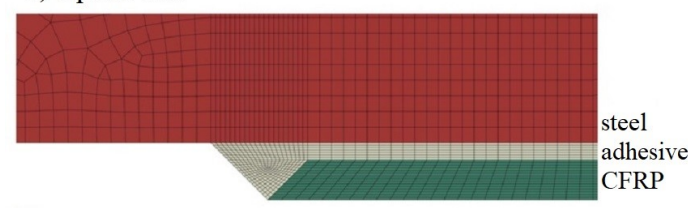

b) reverse tapered end with an adhesive fillet

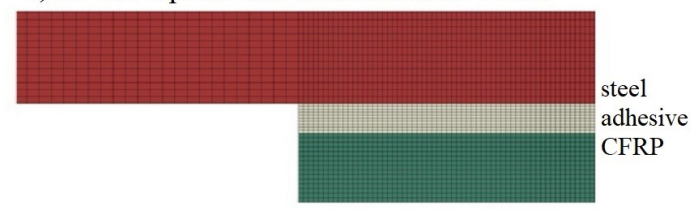

Fig. 4.1. The model of the grid - a section of the reinforcement in the bar with the adhesive strip end area:

a) a reverse-phased end with adhesive outflow,

b) a typical end.

Sample type a):
-- I-beam (60 elements in the flanges and the web heightwise, lengthwise 10 elements per millimetre in the joint end area, normally 0.2 elements per millimetre);

-- adhesive (10 elements heightwise, lengthwise 10 elements per millimetre in the joint end area, normally 0.2 elements per millimetre);

-- strip (10 elements heightwise, lengthwise 10 elements per millimetre in the joint end area, normally 0.2 elements per millimetre);

Sample type b):

-- I-beam (60 elements in the flanges and the web heightwise, lengthwise 5.9 elements per millimetre in the joint end area, normally 0.2 elements per millimetre);

-- I-beam (7-22 elements heightwise, normally 7 elements, lengthwise of 5.9 elements per millimetre in the joint end area, normally 0.2 elements per millimetre); -- strip (15 elements heightwise, lengthwise 5.9 elements per millimetre in the joint end area, normally 0.2 elements per millimetre);

The geometrical model used in the numerical analysis was identical with the one applied in the mathematical analysis and the laboratory tests. The numerical analysis involved bar INP140 with a span of $1.90 \mathrm{~m}$, a reinforced strip $1.4 \mathrm{x} 50 \mathrm{~mm}$ with a length of $1.20 \mathrm{~m}$ with a typical strip end (type "a" in the Fig. 4.1), and a reverse-phased end (45-degree shearing) with an outflow and thickness of the bond end of $0.6 \mathrm{~mm}$ (type "b" in the Fig. 4.1).

As this was the primary verification of the theoretical model, the properties of the reinforced bar within the elastic range was considered, together with the isotropic behaviour of all the materials. The following material properties were assumed: steel - elastic modulus 205GPa, Poisson's coefficient 0.3 ; adhesive - elastic modulus 7.1GPa, Poisson's coefficient 0.35; shear modulus $2.63 \mathrm{GPa}$; composite - elastic modulus $210 \mathrm{GPa}$, Poisson's coefficient 0.3 .

To verify the consistency of the FEM module with the laboratory tests, the strain values on the lower surface of the composite strip were compared.

\subsection{Numerical analysis results}

\subsubsection{The compliance of the FEM model results with the laboratory tests}

It was assumed that the compliance of the numerical module with the laboratory tests would be confirmed if the values of strains measured along with the lower edge of the composite strip had been compliant. To verify the results obtained in the FEM analyses, the values of the calculated strains were confirmed with those identified in the laboratory tests, in points consistent with the measurement points; see Figs. $4.2 \div 4.3$.

The comparison was carried out for bars loaded with two concentrated forces with a value of $20 \mathrm{kN}$. The blue points mark the values of the non-dilatational strains found in the laboratory tests for all the samples (OBT). 
The solid red line marks the outline of the strains identified based on the numerical analysis (FEM). The dotted green line presents the outline of the stresses from the numerical analysis reduced (FEM.R) to the strains obtained in the laboratory measurements.

The lowest square difference error for samples 200.120.ZA was obtained by reducing the numerical analysis value to $102.1 \%$, for samples type 200.120 .FA by reducing the value to $99.8 \%$, and for samples 165.120.FA - to $95.4 \%$ of the value. As a result, it can be concluded that the difference between the values measured and determined using the numerical analysis was $4.6 \%$ (165.120.FA), 2.1\% (200.120.ZA) and 0.2\% (200.120.FA). The value of the difference between the numerical analysis and measurements in this stage makes it possible to conclude that the proper numerical model was assumed for the calculations.

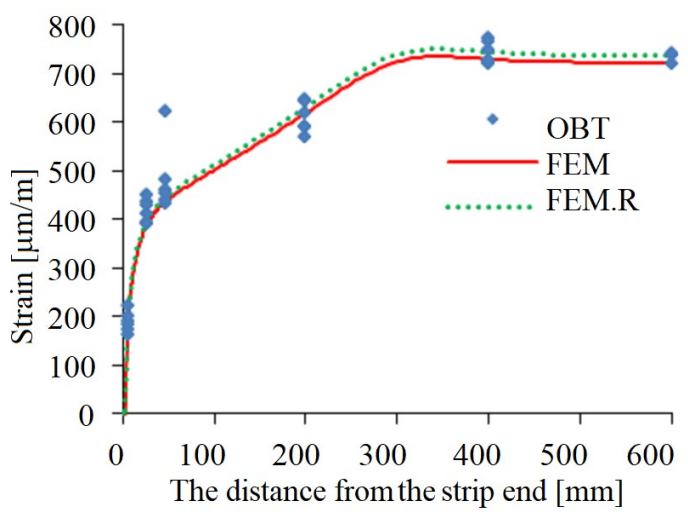

Fig. 4.2. A comparison of the strain diagrams at the lower edge of the composite strip obtained from the FEM analysis and the laboratory tests on sample 200.120.ZA.

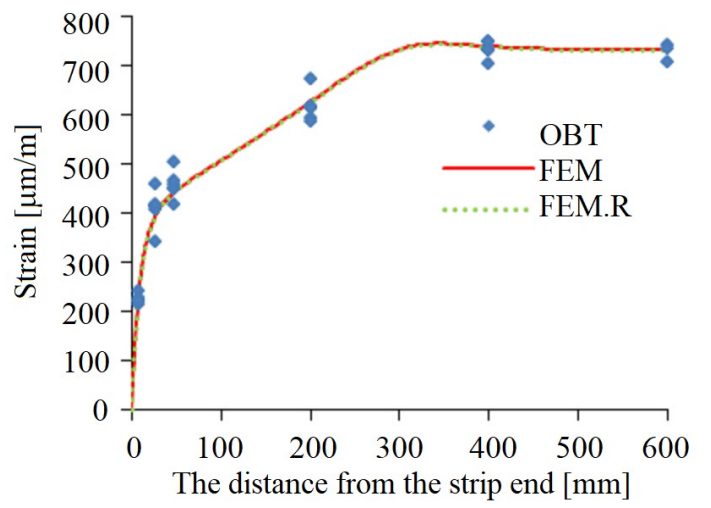

Fig. 4.3. A comparison of the strain diagrams at the lower edge of the composite strip obtained from the FEM analysis and the laboratory tests on sample 200.120.FA.

\subsubsection{Parametric tests}

To verify the results obtained in the laboratory tests, a FEM analysis was carried out on the impact of the following elements on reinforcement: the FRP-laminate thickness, the CFRP strip elastic modulus, thickness of the bond end layer, and the length of the bond. The properties of the material and the load conditions were the same as those applied in the experimental tests.

Based on the numerical analysis results, it was found that using a strip with an elastic modulus of $168 \mathrm{GPa}$, instead of a tape with a modulus of $210 \mathrm{GPa}$, lowers both shearing and peel stresses. The shearing stress value dropped by $12.8 \%$, and the peel stress value by $5.9 \%$. Similar conclusions were drawn from analyses not described in the present study. However, the values of higher destructive forces were obtained for the samples reinforced with a strip with a modulus of $210 \mathrm{GPa}$. Based on the numerical analysis presented in the form of stress diagrams in Figs. $4.4 \& 4.5$, it can be concluded that using a reverse tapered strip with adhesive fillet instead of a strip with a square end has a significant impact - it lowers the values of the shearing and peel stresses. The shearing stress value dropped by $29.4 \%$, and the peel stress value by $71.8 \%$. This leads to an increase in the bearing capacity of the reinforcement system, which complies with the results obtained in the laboratory and analytical tests.

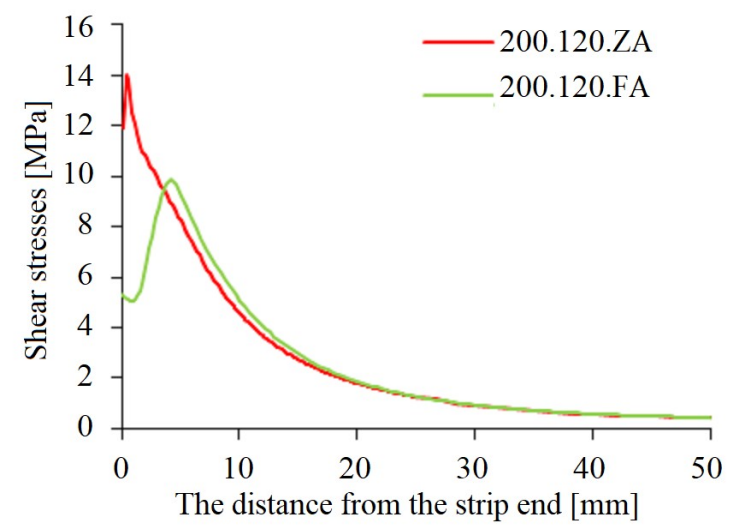

Fig. 4.4. Diagrams of the shearing-stress tests at the bond end of the bar, reinforced with a strip with a length of $1200 \mathrm{~mm}$ with a modulus of $210 \mathrm{GPa}$, thickness of $1.4 \mathrm{~mm}$ with a square end, and a reverse tapered strip with adhesive fillet, with bond end thickness of $0.6 \mathrm{~mm}$.

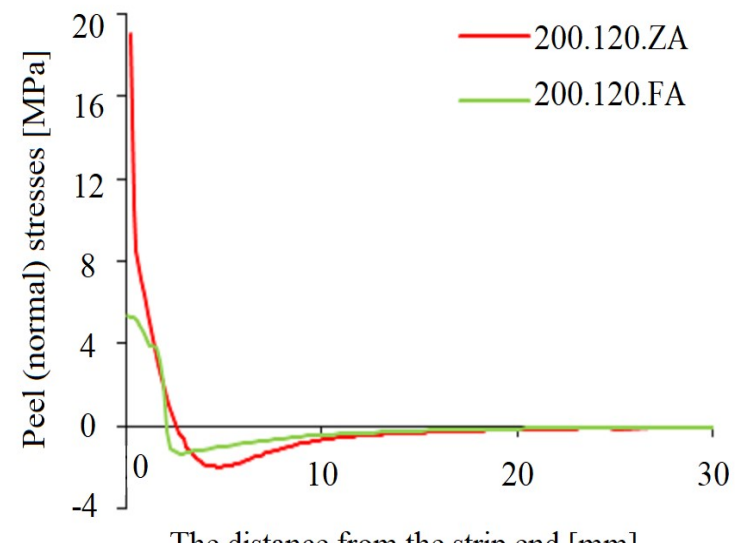

The distance from the strip end [mm]

Fig. 4.5. Diagrams of peel stress tests at the bond end of the bar, reinforced with a strip with a length of $1200 \mathrm{~mm}$ with a modulus of $210 \mathrm{GPa}$, thickness of $1.4 \mathrm{~mm}$ with a square end, and a reverse tapered strip with adhesive fillet, with bond end thickness of $0.6 \mathrm{~mm}$. 
4.2.3 A comparison of stress diagrams from the tensometric measurements and the numerical analysis

Based on the values of shearing stresses obtained from strain measurements in the laboratory tests and from the numerical analyses, the values and the distribution of stresses at the bond end were compared. Figs. 4.6 $\div 4.8$ present the distribution of shearing stresses at the bond end lengthwise after loading the bars with two concentrated forces with a value of $20 \mathrm{kN}$.

The descriptions of the Figures have the following meanings:

OBT - the values of shearing stresses obtained in laboratory tests.

FEM.CA - the value of stresses from the FEM analysis at the strip-adhesive bond.

FEM.SA - the value of stresses from the FEM analysis at the steel-adhesive bond.

THEO - the value of stresses from the analytical analysis at the strip-adhesive bond.

The presence of two types of shearing and peel stresses in the FEM analyses is caused by their difference depending on the contact line.

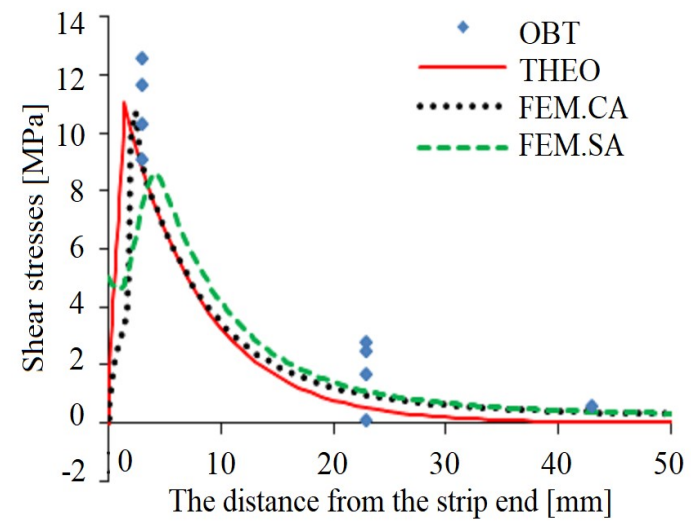

Fig. 4.6. A comparison of the values and distribution of shearing stresses at the bond end of reinforced bar type 165.120.FA.

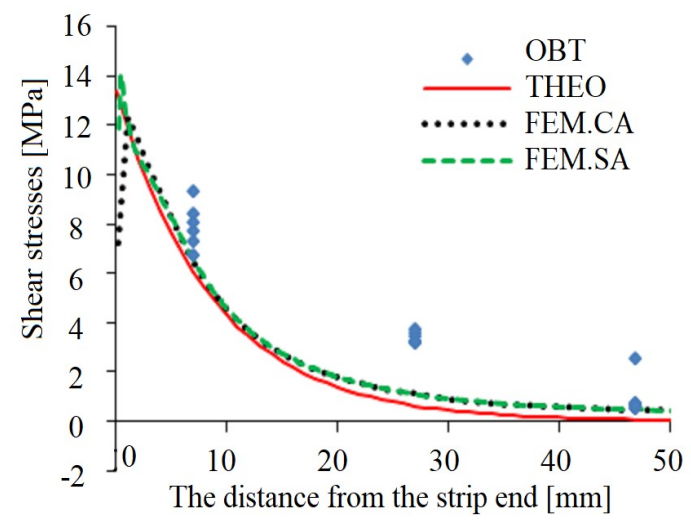

Fig. 4.7. A comparison of the values and distribution of shearing stresses at the bond end of reinforced bar type 200.120.ZA.

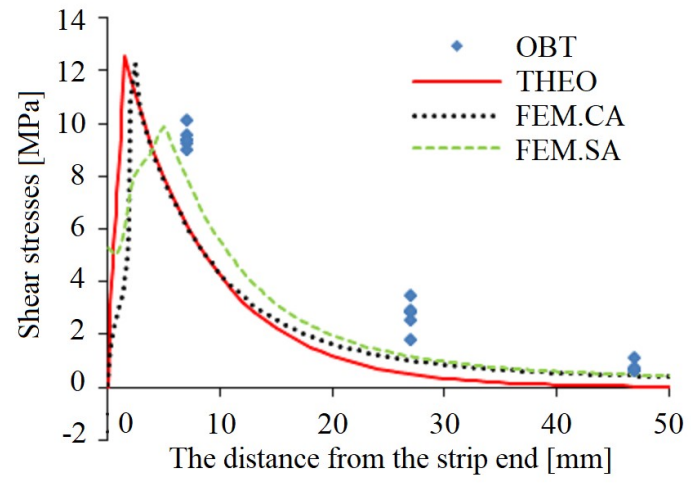

Fig. 4.8. A comparison of the values and distribution of shearing stresses at the bond end of reinforced bar type 200.120.FA.

Analysing Figs. $4.6 \div 4.8$, it can be noted that the diagrams of shearing stresses determined on the basis of the FEM analysis and the maximum values are consistent with the laboratory test results.

\section{Conclusions}

On the basis of the laboratory tests and the numerical analysis, the following conclusions can be drawn.

-- There is an acceptable compliance with the results for the strains measured in the laboratory tests and those determined based on the numerical models. Based on the three types of sample it was found that the differences between the laboratory measurements and the numerical models were below $5 \%$.

-- Shearing stress diagrams were calculated using the numerical method and their maximum values are consistent with the laboratory test results.

-- The change in stress values at bond end thickness is of some significance. The peel stress in the upper edge of the bond end has a positive value, and at the lower edge the value is negative. In fact, the negative stress in the lower edge does not contribute to bond damage. Peeling is a result of positive peel stresses concentrated in the upper edge of the bond end, as described in [25]. Changes in stress values at bond end thickness is more important in the case of peeling stresses.

As the described numerical analyses were of a preliminary nature and were simplified (the elastic range, the isotropic properties of materials) and their main purpose was to verify the analytical model, which is not described in this study, and to compare them with the laboratory test results, future studies should focus on:

-- Developing a model for elastic and plastic properties taking into consideration the anisotropy of adhesive materials (including at least the adhesive and the CFRP strip).

-- Developing models for other types of sample which was not included in the numerical analysis and described in this study (samples with strips with a modulus of $210 \mathrm{GPa}$, a length of $80 \mathrm{~cm}$ and adhesive thickness $0.6 \mathrm{~mm}$, and strips with a length of $100 \mathrm{~cm}$, and bond end 
thickness of $0.6 \mathrm{~mm}, 1.2 \mathrm{~mm}$ and $1.8 \mathrm{~mm}$, all with a reverse tapered strip end with adhesive fillet).

-- A more detailed analysis of the impact of peel stresses on the bearing capacity of the reinforcement system.

\section{References}

1. G. Łagoda, M. Łagoda. 33rd IABSE Symposium, Bangkok, Thailand, Sust. Infrastr. Env. Friendly, Safe and Res. Eff. Vol. 96,September 9-11, 2009.

2. S. Luke. Oxford and Qafco Prill tower, Qatar, NGCC first annual conference and AGM Composites in construction, through life performance, BRE, Watford, UK, 30-31 October 2001.

3. S. Luke. International Conference on FRP Composites in Civil Engineering, J. G. Teng, ed., Hong Kong, China, v. 2.

4. Moy S. S. J., Bloodworth A. G. ICE Proceedings Struct. and Build. 160/SB2 (2007).

5. N. Farmer, I. Smith. Proc. 9th Int'l Conference on Structural Faults and Repairs (2001), 4th - 6th July 2001, London.

6. Church D.G., Silva T.M.D. Proceedings Advanced Polymer Comp. for Struct. Appl. in Constr., Thomas Telford, London 2002.

7. D. Schnerch, K. Stanford, E. Sumner, S. Rizkalla. Proceedings of the International Symposium on Bond Behaviour of FRP in Structures (BBFS 2005) Chen and Teng (eds)(C 2005 International Institute for FRP in Construction 435.
8. D. Schnerch, M. Dawood, S. Rizkalla, E. Sumner, K. Stanford. Adv. in Struct. Eng. 9, 6 (2006).

9. A.F. Harris, A. Beevers. Int. J. of Adh.\& Adh. 19 (1999).

10. D.E. Packham. Int. J. of Adh. \& Adh. 23 (2003) 437-448

11. M. Łagoda, M. Kowal. Bud. i Arch. 12, 2 (2013).

12. M.Y.Tsai, J.Morton. Comp. Struct. 32 (1995).

13. M.D. Fitton, J.G. Broughton. Int. J. of Adh. \& Adh 25 (2005).

14. M. Kowal, M. Łagoda. Struct. and Env. 6, 4 (2014).

15. P.Colombi, C.Poggi. Comp.: Part B 37 (2006).

16. J.Deng, M.M.K.Lee. Comp.Struct.78 (2007).

17. D. Linghoff, M. Al-Emrani, R. Kliger. Comp.: Part B (2009), doi:10.1016/j.compositesb.2009.05.008.

18. N. Ochi, M. Matsumura, N. Hisabe. Proc. Engi. 14 (2011).

19. Y. J. Kim, G. Brunell. Comp. Struct. 93 (2011).

20. M. Kowal, M. Łagoda. R.a.B. - D.i.M. 16, 2 (2017), 85-99, DOI: 10.7409/rabdim.017.006.

21. J.Deng, M.M.K. Lee, S. S.J. Moy. Comp. Struct. 65 (2004).

22. J. Yang, J.F. Chen, J.G. Teng. Constr. and Build. Mat. 23 (2009).

23. M. Bocciarelli, P. Colombi. Eng. Struct. 49 (2013).

24. S.P. Chiew, Y. Yu, C.K. Lee. Comp.: Part B 42 (2011).

25. Y. Yu, S.P. Chiew, C.K. Lee. Comp.: Part B 42 (2011). 